\title{
ANÁLISIS NUMÉRICO DE LOS MAPAS DE PATRONES DE FLUJO DEL REFRIGERANTE R600A EN UN COLECTOR/EVAPORADOR DE UNA BOMBA DE CALOR ASISTIDA POR ENERGÍA SOLAR \\ NUMERICAL ANALYSIS OF FLOW PATTERN MAP OF R600A IN A COLLECTOR/EVAPORATOR OF A SOLAR-ASSISTED HEAT PUMP
}

\author{
William Quitiaquez ${ }^{1, *}$ (D), Eduardo Cortez $^{2}$ (D), Karen Anchapaxi² (D), \\ C.A. Isaza-Roldán ${ }^{3}$ (D), César Nieto-Londoño ${ }^{4}$ (D), Patricio Quitiaquez ${ }^{1}$ (D),
}

Fernando Toapanta-Ramos ${ }^{1}$

Recibido: 30-10-2020, Recibido tras revisión: 26-05-2021, Aceptado: 08-06-2021, Publicado: 01-07-2021

\section{Resumen}

En la presente investigación se detallan los mapas de patrones de flujo que se generan en una tubería horizontal de un colector/evaporador componente de una bomba de calor de expansión directa asistida por energía solar, utilizando el refrigerante R600a como fluido de trabajo. El estudio se realizó en una tubería de $3,8 \mathrm{~mm}$ de diámetro interno y $1000 \mathrm{~mm}$ de longitud, las velocidades de masa variaron entre 197,59 y $267,26 \mathrm{~kg} \cdot \mathrm{m}^{-2} \cdot \mathrm{s}^{-1}$, el flujo de calor entre $72,83 \mathrm{y}$ $488,27 \mathrm{~W} \cdot \mathrm{m}^{-2}$, durante las pruebas experimentales se presentó una radiación solar incidente entre 0 y $652,9 \mathrm{~W} \cdot \mathrm{m}^{-2}$. Se consideró para el análisis la correlación de Wojtan, Ursenbacher y Thome, el modelo utilizado no requiere de cálculos iterativos, además, plantea un análisis detallado de las diferentes zonas presentes a lo largo de la tubería, evidenciando una mayor precisión en los resultados. Las zonas predominantes en los resultados de este estudio son intermitente, anular y secado, encontrados en las cinco pruebas, sin embargo, por las condiciones de trabajo en todas las pruebas a excepción de la prueba A, se encontró el flujo niebla y estratificado-ondulado.

Palabras clave: patrones de flujo, flujo bifásico, transferencia de calor, R600a

\section{Abstract}

This research work presents a detailed description of the flow patterns maps generated in a horizontal pipe of the collector/evaporator of a direct-expansion solar-assisted heat pump, using R600a refrigerant as working fluid. The study was performed in a pipe with an internal diameter of $3.8 \mathrm{~mm}$ and a length of $1000 \mathrm{~mm}$, mass velocities varying between 197.59 and $267.26 \mathrm{~kg} \cdot \mathrm{m}^{-2} \cdot \mathrm{s}^{-1}$ and heat flux between 72.83 and $488.27 \mathrm{~W} \cdot \mathrm{m}^{-2}$; during the experimental tests, an incident solar radiation between 0 and $652.9 \mathrm{~W} \cdot \mathrm{m}^{-2}$ was present. The Wojtan, Ursenbacher and Thome correlation was considered for the analysis and the model used does not require iterative calculations; moreover, it carries out a detailed analysis of the different zones present along the pipe. The predominant zones in this study are intermittent, annular and dryout, found in the five tests, however, due to the working conditions in all tests with the exception of test A, mist and stratified-wavy flow were found.

Keywords: Flow patterns, two-phase flow, heat transfer, R600a

\footnotetext{
$\overline{1, *}$ Carrera de Ingeniería Mecánica - Programa de Maestría en Producción y Operaciones Industriales / GIERIMP, Universidad Politécnica Salesiana, Quito, Ecuador. Autor para correspondencia wquitiaquez@ups.edu.ec. ${ }^{2}$ Carrera de Ingeniería Mecánica, Universidad Politécnica Salesiana, Quito, Ecuador.

${ }^{3}$ Centro de Investigación en Refrigeración y Aire Acondicionado, Universidad Pontificia Bolivariana, Colombia.

${ }^{4}$ Facultad de Ingeniería Aeroespacial, Universidad Pontificia Bolivariana, Colombia.
}

Forma sugerida de citación: Quitiaquez, W.; Cortez, E.; Anchapaxi, K.; Isaza-Roldán, C.A.; Nieto-Londoño, C.; Quitiaquez, P. y Toapanta-Ramos, F. (2021). «Análisis numérico de los mapas de patrones de flujo del refrigerante R600a en un colector/evaporador de una bomba de calor asistida por energía solar». INGENIUS. N. ${ }^{\circ} 26$, (julio-diciembre). pp. 111-121. DOI: https://doi.org/10.17163/ings.n26.2021.10. 


\section{Introducción}

Desde décadas pasadas hasta la actualidad se han instrumentado diferentes protocolos que ayuden a mitigar los problemas ambientales [1,2]. Abas et al. [3] indican que los sistemas de refrigeración se encuentran bajo un periodo de permiso prescrito del Protocolo de Kioto el cual, en conjunto con la Unión Europea y el Acuerdo de París, enfatizaron en la eliminación de refrigerantes que dañen la capa ozono y se propone reemplazarlos por refrigerantes naturales. Por ello alrededor del mundo se realizan investigaciones con los refrigerantes tipo hidrocarburo, como el R290 y R600a debido a que presentan un potencial de agotamiento de ozono (ODP, por sus siglas en inglés) de 0 y un potencial de calentamiento global (GWP, por sus siglas en inglés) de 3 [4].

$\mathrm{Yu}$ et al. [5] utilizan los refrigerantes R290 y R600a como alternativa al R134a. Los resultados indican que los sistemas de refrigeración consumen menos electricidad y la carga del refrigerante es de 30 a $60 \%$ menor con relación a la carga del R134a, llegando a la conclusión de que mejora el factor de energía de los sistemas de refrigeración disminuyendo la carga de refrigerante con el R600a y aliviando aún más las emisiones directas al medioambiente producidas por refrigerantes [6].

Los patrones de flujo predicen la hidrodinámica del flujo y la transferencia de calor que ocurre entre una tubería y el fluido que se transporta dentro de ella, al tener una mayor tasa de transferencia de calor, el refrigerante cambiará rápidamente de fase e ingresará al compresor netamente en la fase de vapor [7]. El régimen de flujo que predice un cambio de fase casi total es el patrón de flujo niebla visualizado a la salida del colector/evaporador, mismo que se presenta en investigaciones como las realizadas por Wojtan et al. [8].

Para determinar los patrones de flujo que se pueden presentar en tuberías horizontales se analiza la correlación de Wojtan et al. [8], debido a que proponen varias modificaciones al mapa propuesto por Kattan et al. [9]. La correlación de Wojtan et al. [8] no presenta cálculos iterativos e indica que la zona estratificada ondulada se subdivide en tres zonas más, siendo bache (slug), slug + estratificada ondulada y estratificada ondulada, además, se menciona un análisis extra en la transición de las zonas de anular a secado y de secado a niebla y a su vez se conoce el inicio y el final de la zona secado que se puede presentar en este tipo de tuberías.

Mashouf et al. [10] realizaron una investigación trabajando con tubos horizontales y el refrigerante R600a; presentaron diferentes patrones de flujo a velocidades de masa entre 114 y $368 \mathrm{~kg} \cdot \mathrm{m}^{-2} \cdot \mathrm{s}^{-1}$, con temperatura de saturación entre 38 y $42{ }^{\circ} \mathrm{C}$ y calidad de 0,8 para el proceso de ebullición del refrigerante. Los resultados obtenidos fueron un flujo intermitente y anular en un tubo con hoyuelos, mientras que, para un tubo liso se observó un flujo intermitente, anular y estratificado ondulado. Así mismo, Vahabi et al. [11] efectuaron un estudio de los patrones de flujo en un tubo de cobre con diámetro interno de 8,7 y $1200 \mathrm{~mm}$ de longitud, con el uso del refrigerante R600a. La prueba fue realizada con valores de velocidad de masa (GA) entre $155 \mathrm{y}$ $470 \mathrm{~kg} \cdot \mathrm{m}^{-2} \cdot \mathrm{s}^{-1}$ con una calidad entre 0,05 y 0,78 , encontrando los flujos: intermitente, anular y estratificado ondulado para el tubo liso.

De Oliveira et al. [12] determinaron la caída de presión y los patrones de flujo al usar el refrigerante R600a, en un tubo horizontal de $1 \mathrm{~mm}$ de diámetro interno. Las condiciones de flujo de calor fluctuaron entre 5 y $60 \mathrm{~kW} \cdot \mathrm{m}^{-2}$, velocidades de masa entre 240 y $480 \mathrm{~kg} \cdot \mathrm{m}^{-2} \cdot \mathrm{s}^{-1}$ y una temperatura de saturación de $25^{\circ} \mathrm{C}$, durante el proceso de ebullición lograron determinar que los flujos predominantes son tapón, slug y anular.

Yang et al. [13] estudiaron los patrones de flujo en dos fases que se pueden presentar en una tubería horizontal de diámetro interno de $6 \mathrm{~mm}$, con el uso del refrigerante R600a. Los valores obtenidos de forma experimental fueron: flujo de calor $(q)$ entre 10,6 y $75 \mathrm{~kW} \cdot \mathrm{m}^{-2}$, velocidades de masa entre $67 \mathrm{y}$ $194 \mathrm{~kg} \cdot \mathrm{m}^{-2} \cdot \mathrm{s}^{-1}$ y presiones de saturación entre 0,215 y $0,415 \mathrm{MPa}$. Utilizando una cámara de alta velocidad, observaron los flujos de tapón, estratificado ondulado, slug y anular.

Zhu et al. [14] investigaron los patrones de flujo durante el proceso de ebullición del refrigerante R32 en minicanales horizontales, trabajando con diámetros internos de 1 y $2 \mathrm{~mm}$, temperaturas de saturación entre 10 y $20{ }^{\circ} \mathrm{C}$, velocidades de masa entre 500 y $600 \mathrm{~kg} \cdot \mathrm{m}^{-2} \cdot \mathrm{s}^{-1}$, flujos de calor entre 10 y $30 \mathrm{~kW} \cdot \mathrm{m}^{-2}$ y calidades de vapor entre 0 y 1 . Los flujos que se observaron fueron slug, anular, secado, niebla y estratificado. Determinaron que la región del flujo slug es pequeña cuando se incrementa la velocidad de la masa y es mayor cuando se incrementa el diámetro del tubo, la temperatura de saturación o el flujo de calor. Para la región anular, es más pequeña cuando aumenta el diámetro del tubo, la temperatura de saturación, el flujo de calor o la velocidad de masa. La región del flujo niebla tiene una mayor presencia cuando incrementan su valor la temperatura de saturación, la velocidad de masa o el flujo de calor y se reduce cuando incrementa el diámetro del tubo.

Zhuang et al. [15] observaron los flujos: tapón, slug, intermitente y anular, donde concluyeron que las calidades de vapor disminuyen con el aumento de la velocidad de la masa, mientras que la variación de la presión de saturación no tiene mayor influencia en las zonas que se puedan presentar en los análisis. Liu et $a l$. [16], en su investigación sobre patrones de flujo, identificaron seis zonas donde el flujo estratificado solo existe cuando la velocidad de la masa es baja, mientras 
que, para la transición del flujo intermitente a anular, dependerá de la velocidad de la masa y el flujo de calor, es decir, si estas aumentan la línea de transición cambiará a una calidad más baja.

De Oliveira et al. [17] estudiaron la transferencia de calor por ebullición utilizando como fluido de trabajo el refrigerante R600a en una tubería horizontal de diámetro interno de $1 \mathrm{~mm}$. Las pruebas se llevaron a cabo usando diferentes rangos de flujo de calor y velocidad de masa de 5 a $60 \mathrm{~kW} \cdot \mathrm{m}^{-2}$ y de 240 a $480 \mathrm{~kg} \cdot \mathrm{m}^{-2} \cdot \mathrm{s}^{-1}$, respectivamente, además de una temperatura de saturación de $25^{\circ} \mathrm{C}$. Los patrones de flujos observados dentro de las pruebas realizadas son anular ondulado, anular liso, slug y tapón, siendo los flujos anulares los predominantes dentro de todas las pruebas experimentales.

Nasr et al. [18] investigaron la transferencia de calor durante la ebullición del R600a en un tubo horizontal con $8,7 \mathrm{~mm}$ de diámetro interior. Las pruebas se realizaron variando los rangos de velocidad de masa y flujo de calor de $130 \mathrm{a} 380 \mathrm{~kg} \cdot \mathrm{m}^{-2} \cdot \mathrm{s}^{-1}$ y de $10 \mathrm{a}$ $27 \mathrm{~kW} \cdot \mathrm{m}^{-2}$. Para valores de calidad hasta 0,7 los patrones de flujo predominantes dentro de todas las pruebas experimentales son: intermitente y anular.

El presente estudio fue desarrollado para investigar los mapas de patrones de flujo en un colector/evaporador sobre el cual incide radiación solar, utilizando como fluido de trabajo el refrigerante R600a durante el proceso de ebullición. Para ello se realizaron cinco pruebas experimentales a diferentes horarios, donde la transición de flujo será determinada basándose en la correlación propuesta por Wojtan et al. [8].

\section{Materiales y métodos}

La combinación de una bomba de calor y energía solar se conoce como un sistema de bomba de calor asistida por energía solar (SAHP, por sus siglas en inglés). Al momento de fusionar el colector y el evaporador en un solo componente, el sistema se convierte en una bomba de calor de expansión directa asistida por energía solar (DX-SAHP, por sus siglas en inglés) [19]. El coeficiente de operación (COP) de los sistemas DXSAHP se encuentra por encima del sistema de bomba de calor convencional, debido a las altas temperaturas generadas por su fuente de energía solar [20]. Los sistemas DX-SAHP tienen un gran potencial en diversas aplicaciones, como calentamiento de espacios, de agua, están conformados por un colector/evaporador, un compresor, un condensador y una válvula de expansión [21].

$\mathrm{Su}$ ciclo de trabajo inicia cuando el refrigerante a baja temperatura y presión ingresa al colector/e- vaporador desde la válvula de expansión, luego se vaporiza al recibir la radiación solar incidente. Al llegar al compresor el fluido se comprime aumentando la temperatura y presión, después de la compresión el vapor fluye hacia el condensador y libera calor hacia el medio que lo contiene [22]. Posteriormente, la válvula de expansión reduce la temperatura y la presión del refrigerante que proviene de la salida del condensador, finalmente, el refrigerante ingresa al colector/evaporador y el proceso se repite. En la Figura 1 se observa un esquema del sistema DX-SAHP construido [23].

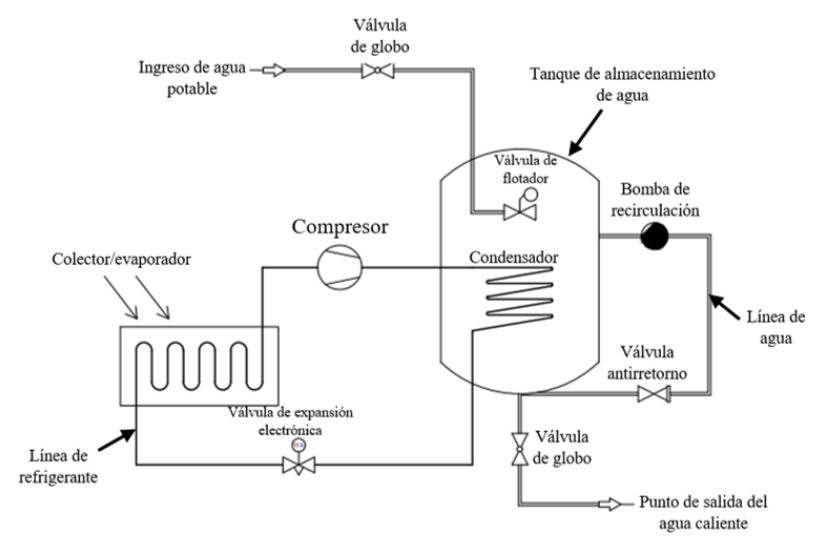

Figura 1. Sistema DX-SAHP propuesto

\subsection{Colector/evaporador}

En la Figura 2 se observa un esquema del colector/evaporador utilizado para el análisis de los mapas de patrones de flujo, del cual se obtuvieron los datos experimentales para proceder con el análisis. Las dimensiones de la tubería horizontal utilizada dentro del colector/evaporador son: diámetro interno de 3,8 $\mathrm{mm}$, longitud de la tubería de $1000 \mathrm{~mm}$, ancho del colector de 223,4 mm y área transversal del colector de $223400 \mathrm{~mm}^{2}$.

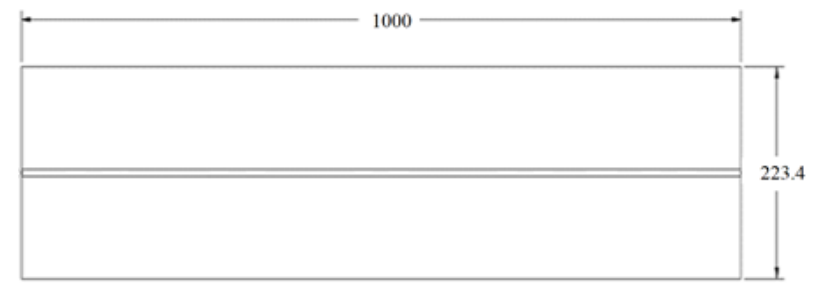

Figura 2. Diagrama esquemático del colector/evaporador $[2]$

Se realizaron pruebas de funcionamiento en diferentes horarios, en la Tabla 1 se presentan los datos experimentales más relevantes. 
Tabla 1. Datos experimentales para 5 pruebas distintas

\begin{tabular}{cccccc}
\hline Variables & Prueba A & Prueba B & Prueba C & Prueba D & Prueba E \\
\hline Hora & $12: 15$ & $12: 40$ & $12: 30$ & $16: 45$ & $20: 40$ \\
Temperatura ambiente $\left[{ }^{\circ} \mathrm{C}\right]$ & 17,6 & 18,3 & 17,9 & 15,4 & 12 \\
Radiación solar incidente & 464,1 & 652,9 & 582,6 & 123,22 & 0 \\
promedio $\left[\mathrm{W} \cdot \mathrm{m}^{-2}\right]$ & 0,00268 & 0,00303 & 0,00295 & 0,0026 & 0,00224 \\
Flujo másico $\left[\mathrm{kg} \cdot \mathrm{s}^{-1}\right]$ & 0,176 & 0,256 & 0,215 & 0,2305 & 0,2325 \\
Calidad de vapor $[-]$ & 14,5 & 14,5 & 14,5 & 12,6 & 9,5 \\
Temperatura superficial $\left[{ }^{\circ} \mathrm{C}\right]$ & & & &
\end{tabular}

2.2. Correlación de Wojtan, Ursenbacher y Thome

A partir del estudio realizado por Wojtan et al. [8] el cual es una actualización de la correlación de Kattan et al. [9] se identifican las ecuaciones para determinar cada una de las zonas que se pueden presentar en el colector/evaporador. La ventaja de utilizar esta correlación es que no presenta ningún cálculo iterativo siendo su estudio confiable y uno de los más actuales e implementados en varias investigaciones como las realizadas por Singh et al. [24] y Yang y Hrnjak [25].

\subsubsection{Fracción de vacío y flujo estratificado}

Wojtan et al. [8] proponen la Ecuación (1) como la forma de obtener la fracción de vacío, mientras que el ángulo estratificado se lo obtiene a partir de la Ecuación (2), además, es necesario conocer la altura del líquido y el perímetro adimensional de la interfaz, mostrados en las Ecuaciones (3) y (4), respectivamente [26].

$$
\begin{gathered}
\alpha=\frac{x}{\rho_{v}}\left\{\begin{array}{c}
{[1+0.12(1-x)]\left(\frac{x}{\rho_{v}}+\frac{1-x}{\rho_{L}}\right) \ldots} \\
\ldots+\frac{1.18(1-x)\left[g \sigma\left(\rho_{L}-\rho_{v}\right)\right]^{0.25}}{G_{A} \rho_{L}^{0.5}}
\end{array}\right\} \\
\theta_{\text {strat }}=2 \pi-2\left\{\begin{array}{c}
\pi(1-\alpha)+\left(\frac{3 \pi}{2}\right)^{\frac{1}{3}} \cdots \\
\ldots\left[1-2(1-\alpha)+(1-\alpha)^{\frac{1}{3}}-\alpha^{\frac{1}{3}}\right] \ldots \\
\ldots-\frac{1}{200}(1-\alpha) \alpha[1-2(1-\alpha)] \cdots \\
\cdots\left[1+4\left[(1-\alpha)^{2}+\alpha^{2}\right]\right]
\end{array}\right\} \\
h_{L d}=0.5\left[1-\cos \left(\frac{2 \pi-\theta_{\text {strat }}}{2}\right)\right] \\
P_{\text {id }}=\sin \left(\frac{2 \pi-\theta_{\text {strat }}}{2}\right)
\end{gathered}
$$

\subsubsection{Flujo estratificado a estratificado ondulado}

Wojtan et al. [8] proponen la Ecuación (5) como el cálculo necesario para obtener el flujo estratificado, donde $G_{\text {strat }}=G_{\text {strat }}\left(x_{I A}\right)$ a $\left(x<x_{I A}\right)$ e indica que el flujo es estratificado cuando $G_{A}<G_{\text {strat }}$.

$$
G_{\text {strat }}=\left[\frac{(226.3)^{2} A_{L d} A_{v d}^{2} \rho_{v}\left(\rho_{L}-\rho_{v}\right) g \mu_{L}}{x^{2}(1-x) \pi^{3}}\right]^{\frac{1}{3}}
$$

\subsubsection{Límite entre el flujo estratificado ondulado a intermitente anular}

La Ecuación (6) indica el análisis necesario para el cálculo en la zona ondulada, indicando que se pueden encontrar tres zonas diferentes, siendo:

Zona slug: $G>G_{\text {wavy }}\left(x_{I A}\right)$

Zona slug/estratificada con ondas:

$G_{\text {strat }}<G_{A}<G_{\text {wavy }}\left(x_{I A}\right.$ y $x<x_{I A}$

Zona estratificada ondulada: $x \geq x_{I A}$

$$
G_{\text {wavy }}=\left\{\begin{array}{c}
\frac{16 A_{v d}^{3} g D \rho_{L} \rho_{v}}{x^{2} \pi^{2}\left[1-\left(2 h_{L d}-1\right)^{2}\right]^{0.5}} \cdots \\
\cdots\left[\frac{\pi^{2}}{25 h_{L d}^{2}}\left(\frac{W e}{F r}\right)_{L}^{-1}+1\right]
\end{array}\right\}^{0.5}+50
$$

\subsubsection{Transición de intermitente a anular}

Wojtan et al. [8], Yang et al. [27], indican que la Ecuación (7) es necesaria para determinar la transición del flujo intermitente a anular.

$$
x_{I A}=\left\{\left[0.34^{\frac{1}{0.875}}\left(\frac{\rho_{v}}{\rho_{L}}\right)^{-\frac{1}{1.75}}\left(\frac{\mu_{v}}{\mu_{L}}\right)^{\frac{1}{7}}\right]+1\right\}^{-1}
$$

\subsubsection{Anular a secado}

La Ecuación 8 muestra el análisis que se debe considerar al momento de determinar la zona secada, propuesta por Wojtan et al. [8] y utilizada por Yang et al. $[27]$.

$G_{\text {dryout }}=\left\{\begin{array}{c}\frac{1}{0.235}\left[\ln \left(\frac{0.58}{x}\right)+0.52\right]\left(\frac{D}{\rho_{v} \sigma}\right)^{-0.17} \ldots \\ \cdots\left[\frac{1}{g D \rho_{v\left(\rho_{L}-\rho_{v}\right)}}\right]^{-0.37} \ldots \\ \cdots\left(\frac{\rho_{v}}{\rho_{L}}\right)^{-0.25}\left(\frac{\dot{q}}{\dot{q}_{\text {crit }}}\right)^{-0.70}\end{array}\right\}^{0.926}$ 


\subsubsection{Secado a niebla}

El cálculo para el cambio entre flujo secado a niebla se determina a partir de la Ecuación (9) propuesta por Wojtan et al. [8].

$$
G_{m i s t}=\left\{\begin{array}{c}
\frac{1}{0.0058}\left[\ln \left(\frac{0.61}{x}\right)+0.57\right]\left(\frac{D}{\rho_{v} \sigma}\right)^{-0.38} \ldots \\
\cdots\left[\frac{1}{g D \rho_{v}\left(\rho_{L}-\rho_{v}\right)}\right]^{-0.15} \ldots \\
\ldots\left(\frac{\rho_{v}}{\rho_{L}}\right)^{0.09}\left(\frac{\dot{q}}{\dot{q}_{c r i t}}\right)^{-0.27}
\end{array}\right\}^{0.926}
$$

Para definir las transiciones en el rango de calidad es necesario conocer las siguientes condiciones [8]:

$$
\begin{aligned}
& \text { Si: } G_{\text {strat }} \geq G_{\text {dryout }} \Rightarrow G_{\text {dryout }}=G_{\text {strat }} \\
& \text { Si: } G_{\text {wavy }} \geq G_{\text {dryout }} \Rightarrow G_{\text {dryout }}=G_{\text {wavy }}
\end{aligned}
$$

Sin embargo, para las ecuaciones de secado y niebla los valores máximos de calidad son 0,99 especificado por Wojtan et al. [8]- [28] y De Oliveira et al. [12]- [29].

\section{Resultados y discusión}

A partir de datos experimentales y la correlación de Wojtan et al. [8], se obtienen los mapas de patrones de flujo correspondientes a cada una de las pruebas realizadas en diferentes horarios.

Cada una de las zonas presentes en los mapas de patrones de flujo se identifican aplicando la siguiente nomenclatura: Intermitente (I), Anular (A), Estratificado (S), Estratificado ondulado (SW), Secado (D) y Niebla (M). Conforme el refrigerante circula a lo largo de la tubería del colector/evaporador, su calidad aumenta, dando lugar a la presencia de los diferentes patrones de flujo.

\subsection{Mapas de patrones de flujo en diferentes pruebas experimentales}

\subsubsection{Mapa de patrones de flujo de la prueba A}

En la Figura 3 se observa el mapa de patrón de flujo obtenido a las 12:15.

La velocidad de masa de $236,5 \mathrm{~kg} \cdot \mathrm{m}^{-2} \cdot \mathrm{s}^{-1}$ indica que las zonas presentes en esta tubería del colector/evaporador, son intermitente, anular y secado, con rangos de calidad de 0,176-0,2686, 0,2686-0,9484 y 0,9484 0,99 , respectivamente. Se muestra un rango de visualización mayor en la zona anular, mientras que la zona secada es la que menos visualización presenta.

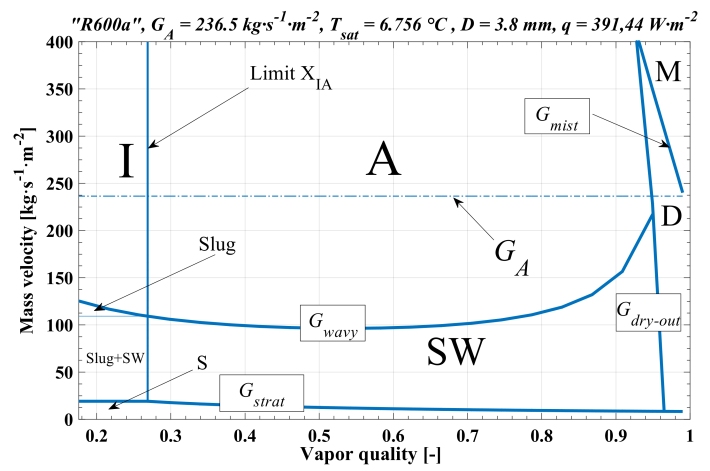

Figura 3. Mapa de patrones de flujo de la prueba A para el refrigerante $\mathrm{R} 600 \mathrm{a}$ con una $\mathrm{T}_{\text {sat }}=6,756{ }^{\circ} \mathrm{C}$, $\mathrm{D}=3,8 \mathrm{~mm}, \mathrm{q}=391,44 \mathrm{~W} \cdot \mathrm{m}^{-2}$ y $\mathrm{G}_{\mathrm{A}}=236,5 \mathrm{~kg} \cdot \mathrm{m}^{-2} \cdot \mathrm{s}^{-1}$

\subsubsection{Mapa de patrones de flujo de la prueba $\mathrm{B}$}

La prueba B se la realizó a las 12:40, obteniendo los patrones de flujo intermitente, anular, secado y niebla, los cuales se visualizan en la Figura 4. En comparación con la prueba $\mathrm{A}$, se presenta una nueva zona con una velocidad de masa de $267,26 \mathrm{~kg} \cdot \mathrm{m}^{-2} \cdot \mathrm{s}^{-1}$ y con rangos de calidades de 0,256-0,283, 0,283-0,941, 0,980,979 y $0,979-0,99$, respectivamente a las cuatro zonas presentes.

La zona con mayor visualización es la anular, por el rango extendido de calidad, mientras que la zona intermitente es aquella que presenta menor visualización debido a su pequeño rango de calidad.

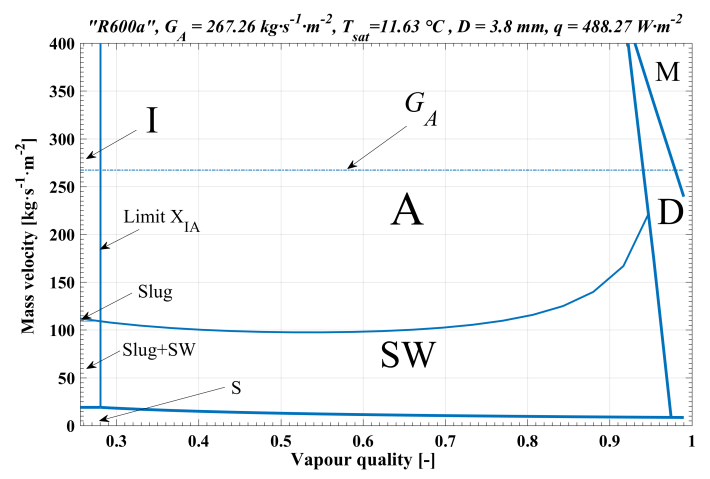

Figura 4. Mapa de patrones de flujo de la prueba B para el refrigerante $\mathrm{R} 600 \mathrm{a}$ con una $\mathrm{T}_{\text {sat }}=11,63{ }^{\circ} \mathrm{C}$, $\mathrm{D}=3,8 \mathrm{~mm}, \mathrm{q}=488,27 \mathrm{~W} \cdot \mathrm{m}^{-2}$ y $\mathrm{G}_{\mathrm{A}}=267,26 \mathrm{~kg} \cdot \mathrm{m}^{-2} \cdot \mathrm{s}^{-1}$

\subsubsection{Mapa de patrones de flujo de la prueba $\mathrm{C}$}

La Figura 5 muestra los patrones de flujo de la prueba C a partir de los datos obtenidos a las 12:30, con una velocidad de masa de $260,115 \mathrm{~kg} \cdot \mathrm{m}^{-2} \cdot \mathrm{s}^{-1}$. Se presentan cuatro zonas: intermitente, anular, secado y 
niebla, ubicadas en rangos de calidad de 0,215-0,275, 0,275-0,943, 0,943-0,982 y 0,982-0,99, respectivamente.

Tanto en la prueba A como en B, la zona con mayor visualización es la anular y el flujo intermitente presenta un rango pequeño como la zona secada.

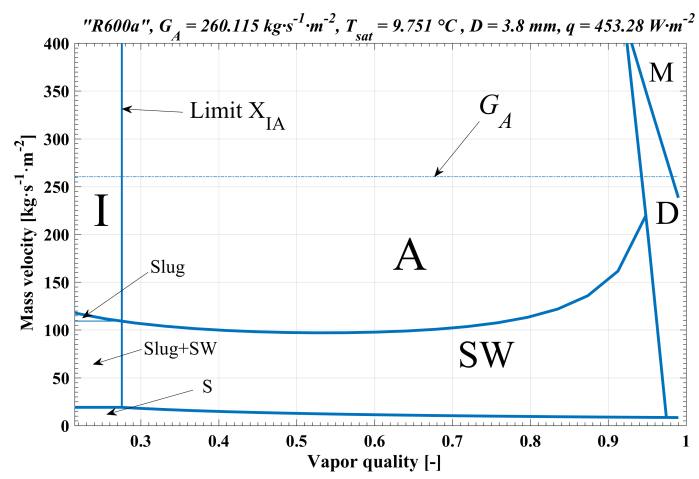

Figura 5. Mapa de patrones de flujo de la prueba $\mathrm{C}$ para el refrigerante $\mathrm{R} 600 \mathrm{a}$ con una $\mathrm{T}_{\text {sat }}=9,751{ }^{\circ} \mathrm{C}$, $\mathrm{D}=3,8 \mathrm{~mm}, \mathrm{q}=453,28 \mathrm{~W} \cdot \mathrm{m}^{-2}$ y $\mathrm{G}_{\mathrm{A}}=260,115$ $\mathrm{kg} \cdot \mathrm{m}^{-2} \cdot \mathrm{s}^{-1}$

\subsubsection{Mapa de patrones de flujo de la prueba $\mathbf{D}$}

El mapa de patrones de flujo obtenido a partir de los datos experimentales tomados a las 16:45, con una velocidad de masa de $229,43 \mathrm{~kg} \cdot \mathrm{m}^{-2} \cdot \mathrm{s}^{-1}$ presenta las zonas intermitente, anular, estratificado ondulado y secado, tal como lo indica la Figura 6.

Los rangos de calidades presentes según los flujos son 0,231-0,267, 0,267-0,952, 0,952-0,961 y 0,961-0,99, respectivamente. La zona que presenta una mayor visualización es la anular, por su rango de calidad, mientras que las demás zonas se presentan en rangos menores de calidad. Las condiciones de trabajo son distintas, no solo la velocidad de masa también influye, el tiempo de toma de datos, por ello se presenta una nueva zona, la estratificada ondulada.

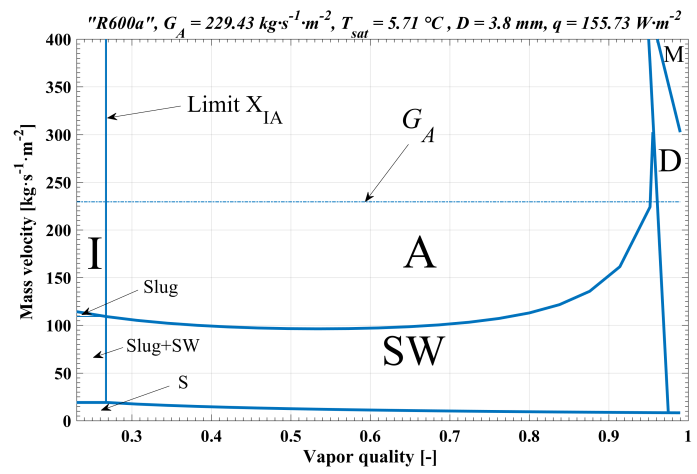

Figura 6. Mapa de patrones de flujo de la prueba D para el refrigerante $\mathrm{R} 600 \mathrm{a}$ con una $\mathrm{T}_{\text {sat }}=5,71^{\circ} \mathrm{C}, \mathrm{D}=3,8 \mathrm{~mm}$, $\mathrm{q}=155,73 \mathrm{~W} \cdot \mathrm{m}^{-2}$ y $\mathrm{G}_{\mathrm{A}}=229,43 \mathrm{~kg} \cdot \mathrm{m}^{-2} \cdot \mathrm{s}^{-1}$

\subsubsection{Mapa de patrones de flujo de la prueba $\mathbf{E}$}

La Figura 7 indica el mapa de patrones de flujo de la prueba E, obtenido a partir de los datos experimentales tomados a las 20:40, donde con una velocidad de masa de $197,59 \mathrm{~kg} \cdot \mathrm{m}^{-2} \cdot \mathrm{s}^{-1}$, los flujos que se presentan en esta prueba son intermitente, anular, estratificado ondulado y secado.

El rango que presenta una mayor visualización es el de la zona anular, mientras que las zonas intermitente y secado son más pequeñas. Como la prueba D, al tener una velocidad de masa menor a las anteriores, se presenta la zona estratificada ondulada.

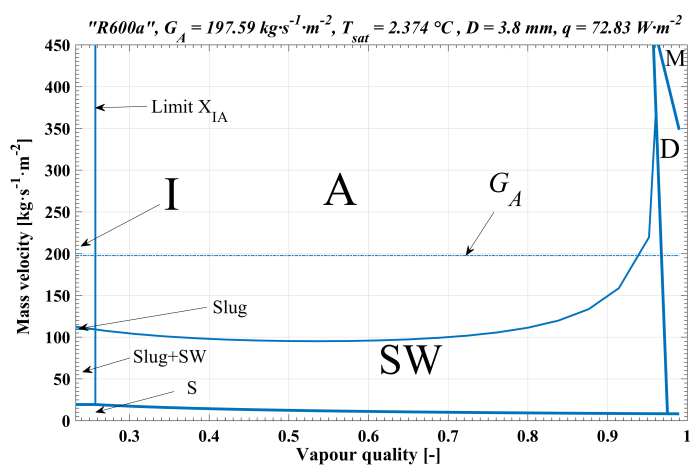

Figura 7. Mapa de patrones de flujo de la prueba E para el refrigerante $\mathrm{R} 600 \mathrm{a}$ con una $\mathrm{T}_{\mathrm{sat}}=2,374{ }^{\circ} \mathrm{C}$, $\mathrm{D}=3,8 \mathrm{~mm}, \mathrm{q}=72,83 \mathrm{~W} \cdot \mathrm{m}^{-2}$ y $\mathrm{G}_{\mathrm{A}}=197,59 \mathrm{~kg} \cdot \mathrm{m}^{-2} \cdot \mathrm{s}^{-1}$

Todas las pruebas indican que los patrones de flujo varían dependiendo de las condiciones de trabajo, por ello, se determina que a medida que la velocidad de la masa es mayor, tiende a estar presente el flujo niebla, mientras que si esta disminuye se puede presentar la zona estratificada ondulada. Además, de las zonas presentes en las cinco pruebas, la de mejor visualización y con mayor rango de calidad viene a ser el flujo anular, siendo aquella que predomina en este estudio, al igual que las zonas intermitente y secado, pero con un menor rango de calidad.

\subsection{Comparación de los patrones de flujo en diferentes horarios}

A continuación, se realiza un análisis en dependencia de los horarios y los resultados obtenidos en los patrones de flujo, así como la diferencia de sus condiciones de trabajo.

\subsubsection{Comparación de la prueba A con la prueba B}

La Figura 8 presenta la comparación de resultados de la prueba A con horario de 12:15 y de la prueba B con horario de 12:40. La radiación solar incidente es de 464,1 y $652,9 \mathrm{~W} \cdot \mathrm{m}^{-2}$ y la velocidad de masa es de $236,5 \mathrm{y} 267,26 \mathrm{~kg} \cdot \mathrm{m}^{-2} \cdot \mathrm{s}^{-1}$, respectivamente. Se puede 
observar que los límites del flujo anular con el estratificado ondulado y niebla, no presentan una variación, mientras que en el límite de la zona secada ya existe un pequeño desfase, así como el límite intermitente anular, en el cual sus calidades iniciales varían de 0,176 a 0,256 .

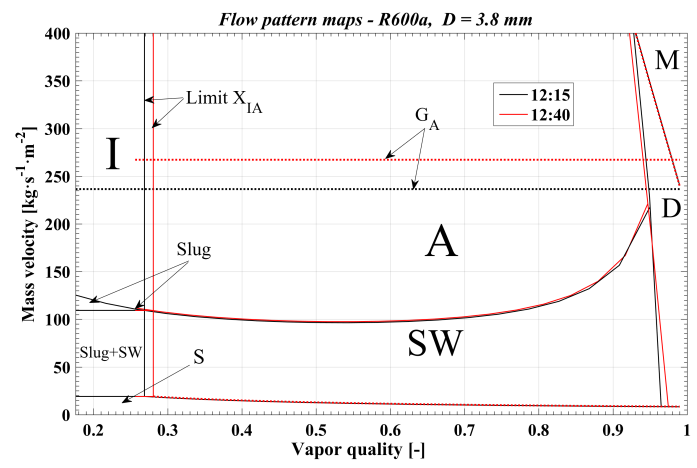

Figura 8. Comparación de los mapas de las pruebas A y B en horarios de 12:15 y 12:40, respectivamente

\subsubsection{Comparación de la prueba A con la prueba D}

La Figura 9 presenta una comparación entre las pruebas A y D, con datos tomados en los horarios de 12:15 y 16:45, radiación solar incidente de 391,44 y $155,73 \mathrm{~W} \cdot \mathrm{m}^{-2}$ y velocidades de masa de 236,5 y 229,43 $\mathrm{kg} \cdot \mathrm{m}^{-2} \cdot \mathrm{s}^{-1}$, respectivamente. Los límites de estratificado y ondulado no presentan ninguna variación, lo cual para los demás límites no es el caso.

En el caso de la prueba B, la velocidad de masa no indica que se presente el flujo estratificado ondulado en esa prueba, sin embargo, en la prueba D, la velocidad de masa es menor y muestra la zona estratificada ondulada. Mientras que los límites de las zonas secado y niebla de la prueba $\mathrm{D}$ se mueven a la derecha por el incremento de la radiación solar incidente.

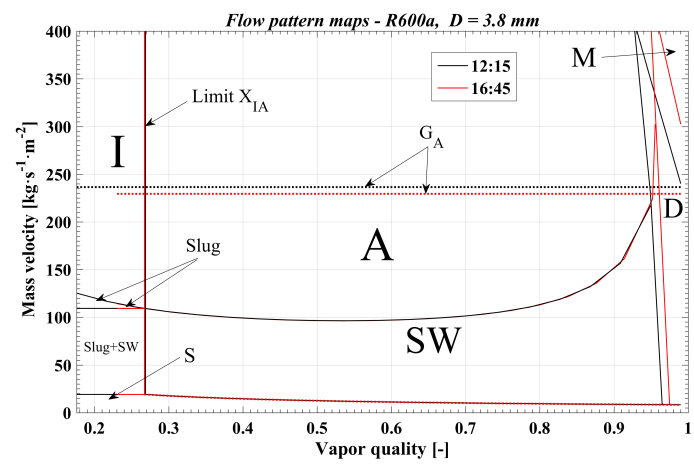

Figura 9. Comparación de los mapas de las pruebas A y D en horarios de 12:15 y 16:45, respectivamente

\subsubsection{Comparación de la prueba D con la prueba E}

La siguiente comparación se muestra en la Figura 10, se comparan las pruebas D y E, con toma de datos experimentales a las 16:45 y 20:40, radiación solar incidente de 123,22 y $0 \mathrm{~W} \cdot \mathrm{m}^{-2}$ y velocidades de masa de 229,43 y $197,59 \mathrm{~kg} \cdot \mathrm{m}^{-2} \cdot \mathrm{s}^{-1}$, respectivamente. Los límites de la zona estratificada ondulada no muestran variación, mientras que, para los límites de la zona secada y niebla, muestran una tendencia a la derecha cuando disminuye la radiación solar incidente en valores de calidad altos. Ambas pruebas indican la presencia de la zona estratificada ondulada con los valores de velocidad de masa mencionados, sin embargo, en la prueba D se logra una mejor visualización del flujo debido a que la velocidad de masa disminuye al ser la radiación solar incidente $0 \mathrm{~W} \cdot \mathrm{m}^{-2}$.

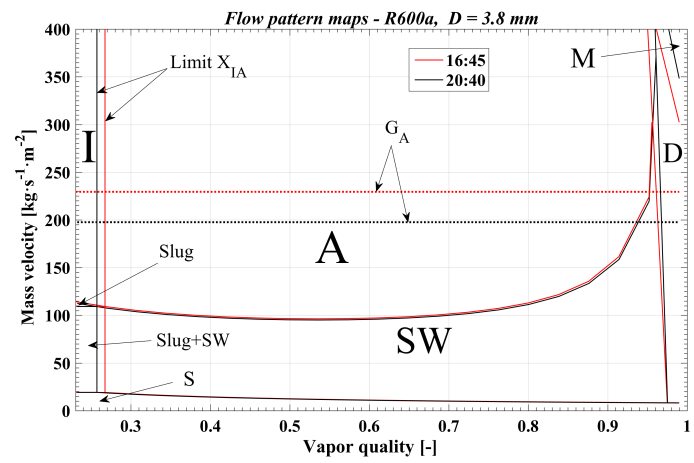

Figura 10. Comparación de los mapas de las pruebas D y E en horarios de 16:45 y 20:40, respectivamente

\subsubsection{Comparación de la prueba A con la prueba E}

En la Figura 11 se observa la comparación de los mapas de patrones de flujo entre la prueba $\mathrm{A}$ y E, con horarios de trabajo de 12:15 y 20:40, radiación solar incidente de 464,1 y $0 \mathrm{~W} \cdot \mathrm{m}^{-2}$ y velocidad de la masa de 236,5 y $197,59 \mathrm{~kg} \cdot \mathrm{m}^{-2} \cdot \mathrm{s}^{-1}$. El límite estratificado ondulado no presenta variación, mientras que los límites secado y niebla se mueven a la derecha en comparación de los límites resultantes del mediodía. La diferencia entre la prueba A y E al presentar las velocidades de masa antes mencionadas, indica que solo la prueba E tiene el flujo estratificado ondulado, mientras que la prueba A no.

\subsubsection{Comparación de las pruebas $\mathrm{A}, \mathrm{D}$ y $\mathrm{E}$}

En la Figura 12 se presenta una comparación entre las pruebas A, D y E con horarios de trabajo de 12:15, 16:45 y 20:40, con radiación solar incidente de 464,1, 123,22 y $0 \mathrm{~W} \cdot \mathrm{m}^{-2}$, respectivamente. Se analiza que la velocidad de masa en la gráfica es directamente proporcional a la radiación solar. 


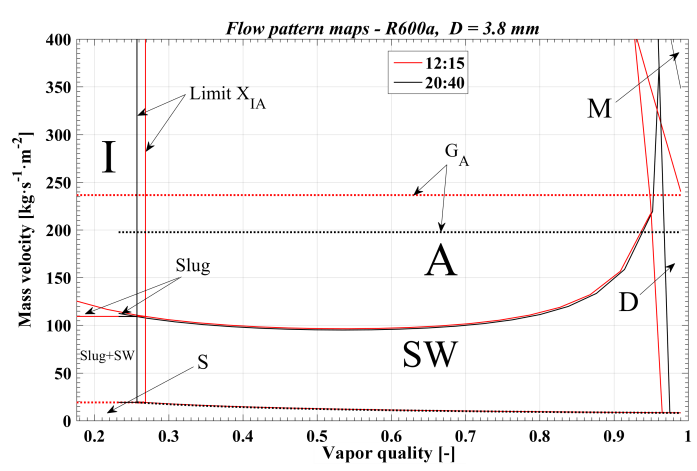

Figura 11. Comparación de los mapas de las pruebas A y $\mathrm{E}$

$\mathrm{Al}$ reducir ambas variables, las zonas secada y niebla se prolongan a la derecha con valores de calidad altos, mientras que los límites de estratificado ondulado no sufren variación. Se indica cómo el límite $x_{I A}$ varía en el inicio de las calidades. Con la velocidad de la masa se pueden identificar las zonas que se presenten en la tubería de acuerdo con cada una de las condiciones, si es más alta será el caso de la prueba A y si es más bajo el caso de la prueba D y E. Las zonas presentes en la prueba A son: intermitente, anular y secado, mientras que para la prueba D y E las zonas presentes son intermitente, anular, estratificado ondulado y secado.

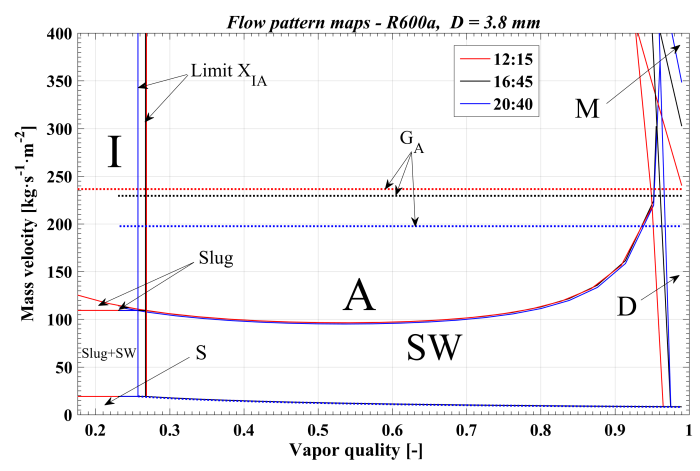

Figura 12. Comparación de los mapas de patrón de flujo con radiación solar incidente a distintos horarios

En los diferentes mapas obtenidos, la prueba A muestra los mejores resultados al tener una calidad inicial menor, los límites dentro de la gráfica observados en la Figura 3, se asemejan al modelo de Wojtan et al. [8] considerado para el análisis del fluido en ebullición. Además, una de las condiciones predominantes dentro de un sistema DX-SAHP es tener a la salida del colector/evaporador un fluido de trabajo en fase de vapor, los patrones de flujo que se acomodan a esta condición son: secado y niebla, los cuales se presentan en mayores rangos de calidad de 0,941-0,99 para la prueba B observada en la Figura 4.

En la Figura 13 se evidencia la relación entre los patrones de flujo y el coeficiente de transferencia de calor (HTC), los patrones de flujo fundamentan su importancia al indicar el cambio de fase de un refrigerante que fluye a través de una tubería. La figura mencionada muestra las zonas: intermitente, anular, secado y niebla. Para los patrones de flujo como intermitente y anular el comportamiento del coeficiente de transferencia de calor presenta una tendencia creciente; al iniciar la zona de secado el HTC tiende a decrecer con el incremento de la calidad de vapor [8]. El fluido de trabajo alcanza un balance calórico cuasi total con la tubería y al llegar a la zona niebla cambia su fase de líquido a vapor completamente, por lo que, el HTC en las zonas secado y niebla tiende a descender a valores mínimos de $516,37 \mathrm{~W} \cdot \mathrm{m}^{-2} \cdot \mathrm{K}^{-1}$, para el ejemplo mostrado.

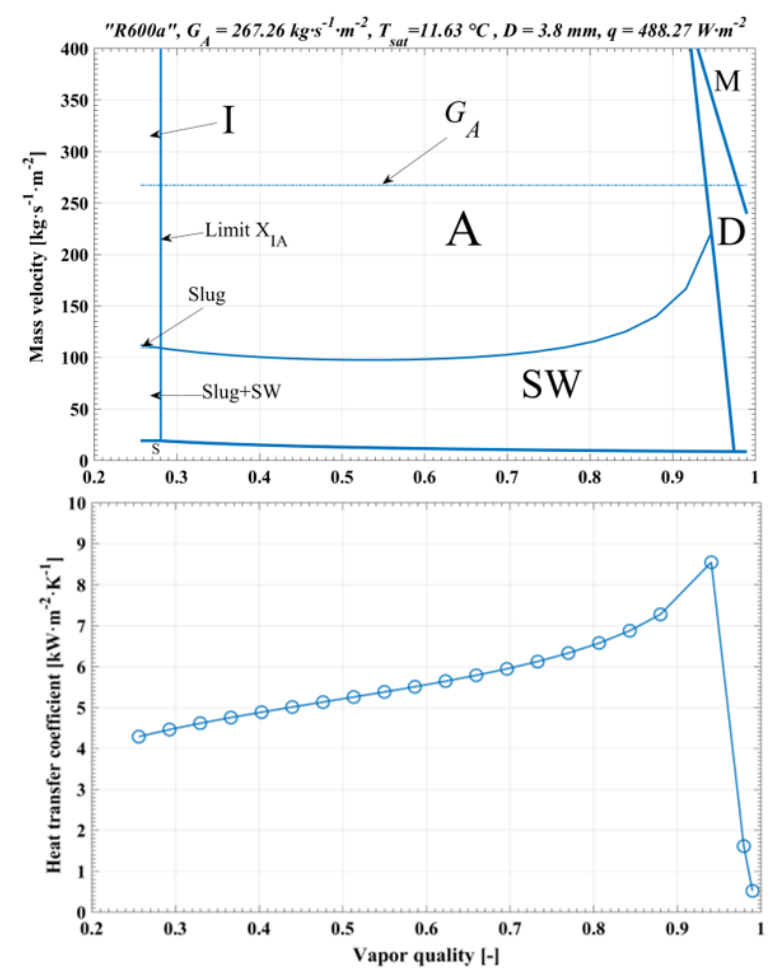

Figura 13. Contrastación de los mapas de patrones de flujo vs. coeficiente de transferencia de calor bajo el modelo matemático de Wojtan et al. [8], [28]

\section{Conclusiones}

Los mapas de patrones de flujo obtenidos en las pruebas experimentales realizadas representan el comportamiento del cambio de fase del refrigerante R600a como respuesta a la energía solar ganada en el sistema. Las gráficas y los límites de los diferentes regímenes de flujo presentaron una variación a partir de los diferentes parámetros de trabajo como: velocidad de masa, radiación solar incidente, flujo de calor, flujo másico [23] y calidad inicial del fluido de trabajo. Así 
mismo, los mapas de patrones de flujo permiten estudiar las distintas configuraciones de distribución de dos fases en un fluido e indican que zona predice el cambio de fase casi total del mismo, para ello se obtuvieron las siguientes conclusiones:

Las zonas predominantes en este estudio son intermitente, anular y secado, al ser los flujos que se presentan en todas las pruebas y las zonas secado y niebla, presentes en diferentes ocasiones. Tal es el caso de la zona niebla, la cual estará presente cuando el flujo de calor y velocidad de masa sean de $488,27 \mathrm{~W} \cdot \mathrm{m}^{-2}$ y $267,26 \mathrm{~kg} \cdot \mathrm{m}^{-2} \cdot \mathrm{s}^{-1}$, respectivamente, mientras que, para la zona estratificada ondulada el flujo de calor y la velocidad de masa serán $72,83 \mathrm{~W} \cdot \mathrm{m}^{-2}$ y 197,59 $\mathrm{kg} \cdot \mathrm{m}^{-2} \cdot \mathrm{s}^{-1}$, respectivamente.

La zona estratificada ondulada se presentó en las pruebas D y E, en rangos de calidad entre 0,952-0,961 y 0,939-0,968 respectivamente. Mientras que, la zona niebla se presenta en la prueba $\mathrm{B}$ con rangos de calidad de 0,979-0,99 y en la prueba $\mathrm{C}$ con rangos de calidad de 0,982-0,99. Además, las pruebas D y E se obtuvieron con valores de radiación solar incidente de 123,22 y $0 \mathrm{~W} \cdot \mathrm{m}^{-2}$, respectivamente.

La prueba A al tener una calidad inicial de 0,176 se asemeja más al modelo de mapa de patrón de flujo tomado como base para este estudio. Sin embargo, en las pruebas B y C se visualiza la mayor zona de secado y niebla, cumpliendo con la condición de que un fluido debe ingresar en fase vapor al compresor.

La presente investigación se realizó utilizando un refrigerante amigable con el medioambiente como el isobutano R600a que presenta un ODP de 0 y un GWP de 3, además su uso presenta mayores conductividades térmicas con referencia a los refrigerantes convencionales como el R22 y el R134a.

\section{Agradecimientos}

Los autores agradecen al Grupo de Investigación en Energías Renovables e Implementación Mecánica de PYMES de la Universidad Politécnica Salesiana y al Grupo de Investigación Energía y Termodinámica de la Universidad Pontificia Bolivariana de Medellín.

\section{Referencias}

[1] J. Calle-Sigüencia and O. Tinoco-Gómez, "Obtención de ACS con energía solar en el cantón Cuenca y análisis de la contaminación ambiental," Ingenius, no. 19, pp. 89-101, 2018. [Online]. Available: https://doi.org/10.17163/ings.n19.2018.09

[2] W. Quitiaquez, J. Estupiñán Campos, C. Isaza Roldán, F. Toapanta-Ramos, and A. Lobato-Campoverde, "Análisis numérico de un sistema de calentamiento de agua utilizando un colector solar de placa plana," Ingenius, no. 24, pp. 97-106, 2020. [Online]. Available: https://doi.org/10.17163/ings.n24.2020.10

[3] N. Abas, A. R. Kalair, N. Khan, A. Haider, Z. Saleem, and M. S. Saleem, "Natural and synthetic refrigerants, global warming: A review," Renewable and Sustainable Energy Reviews, vol. 90, pp. 557-569, 2018. [Online]. Available: https://doi.org/10.1016/j.rser.2018.03.099

[4] K. Nawaz, B. Shen, A. Elatar, V. Baxter, and O. Abdelaziz, "R290 (propane) and R600a (isobutane) as natural refrigerants for residential heat pump water heaters," $A p$ plied Thermal Engineering, vol. 127, pp. 870-883, 2017. [Online]. Available: https: //doi.org/10.1016/j.applthermaleng.2017.08.080

[5] C.-C. Yu and T.-P. Teng, "Retrofit assessment of refrigerator using hydrocarbon refrigerants," Applied Thermal Engineering, vol. 66, no. 1, pp. 507-518, 2014. [Online]. Available: https: //doi.org/10.1016/j.applthermaleng.2014.02.050

[6] X. Zhuang, M. Gong, X. Zou, G. Chen, and J. Wu, "Experimental investigation on flow condensation heat transfer and pressure drop of R170 in a horizontal tube," International Journal of Refrigeration, vol. 66, pp. 105-120, 2016. [Online]. Available: https://doi.org/10.1016/j.ijrefrig.2016.02.010

[7] J. C. Pérez Angulo, C. Simancas, and N. Santos Santos, "Modelamiento y simulación de un sistema no convencional de levantamiento multifasico," Fuentes, el reventón energético, vol. 14, no. 1, pp. 19-34, 2016. [Online]. Available: https://doi.org/10.18273/revfue.v14n1-2016002

[8] L. Wojtan, T. Ursenbacher, and J. R. Thome, "Investigation of flow boiling in horizontal tubes: Part I-A new diabatic two-phase flow pattern map," International Journal of Heat and Mass Transfer, vol. 48, no. 14, pp. 2955-2969, 2005. [Online]. Available: https://doi.org/10.1016/j. ijheatmasstransfer.2004.12.012

[9] N. Kattan, J. R. Thome, and D. Favrat, "Flow boiling in horizontal tubes: Part 1-Development of a diabatic two-phase flow pattern map," Journal of Heat Transfer, vol. 120, no. 1, pp. 140-147, Oct. 1998. [Online]. Available: https://doi.org/10.1115/1.2830037

[10] H. Mashouf, M. Shafaee, A. Sarmadian, and S. Mohseni, "Visual study of flow patterns during evaporation and condensation of R-600a inside horizontal smooth and helically dimpled tubes," Applied Thermal Engineering, vol. 124, pp. 1392-1400, 2017. [Online]. Available: https: //doi.org/10.1016/j.applthermaleng.2017.06.125 
[11] A. Vahabi, M. Shafaee, A. Sarmadian, and H. Mashoof, "Discovering an empirically new relation and obtain the flow pattern map for dimpled tubes in two-phase flow for refrigerant R600-a," Modares Mechanical Engineering, vol. 17, no. 7, 2017. [Online]. Available: http://dorl.net/dor/20. 1001.1.10275940.1396.17.7.25.7

[12] J. D. de Oliveira, J. B. Copetti, and J. C. Passos, "Experimental investigation on flow boiling pressure drop of R-290 and R-600a in a horizontal small tube," International Journal of Refrigeration, vol. 84, pp. 165-180, 2017. [Online]. Available: https://doi.org/10.1016/j.ijrefrig.2017.08.004

[13] Z.-Q. Yang, M. Gong, G. Chen, Z. Lin, H. Huang, and H. Feng, "A new diabatic two phase flow pattern transition model of R600a," International Journal of Refrigeration, vol. 99, pp. 138-144, 2019. [Online]. Available: https://doi.org/10.1016/j.ijrefrig.2018.12.025

[14] Y. Zhu, X. Wu, and R. Zhao, "R32 flow boiling in horizontal mini channels: Part I. Two-phase flow patterns," International Journal of Heat and Mass Transfer, vol. 115, pp. 1223-1232, 2017. [Online]. Available: https://doi.org/10.1016/ j.ijheatmasstransfer.2017.07.101

[15] X. Zhuang, M. Gong, G. Chen, X. Zou, and J. Shen, "Two-phase flow pattern map for R170 in a horizontal smooth tube," International Journal of Heat and Mass Transfer, vol. 102, pp. 1141-1149, 2016. [Online]. Available: https://doi. org/10.1016/j.ijheatmasstransfer.2016.06.094

[16] J. Liu, J. Liu, and X. Xu, "Diabatic visualization study of R245fa two phase flow pattern characteristics in horizontal smooth and microfin tube," International Journal of Heat and Mass Transfer, vol. 152, p. 119513, 2020. [Online]. Available: https://doi.org/10.1016/j. ijheatmasstransfer.2020.119513

[17] J. Diehl de Oliveira, J. Biancon Copetti, and J. C. Passos, "An experimental investigation on flow boiling heat transfer of R-600a in a horizontal small tube," International Journal of Refrigeration, vol. 72, pp. 97-110, 2016. [Online]. Available: https://doi.org/10.1016/j.ijrefrig.2016.08.001

[18] M. Nasr, M. Akhavan-Behabadi, M. Momenifar, and P. Hanafizadeh, "Heat transfer characteristic of r-600a during flow boiling inside horizontal plain tube," International Communications in Heat and Mass Transfer, vol. 66, pp. 93-99, 2015. [Online]. Available: https://doi.org/10.1016/ j.icheatmasstransfer.2015.05.024
[19] Z. Wang, P. Guo, H. Zhang, W. Yang, and S. Mei, "Comprehensive review on the development of sahp for domestic hot water," Renewable and Sustainable Energy Reviews, vol. 72 , pp. 871-881, 2017. [Online]. Available: https://doi.org/10.1016/j.rser.2017.01.127

[20] D. Zhang, Q. Wu, J. Li, and X. Kong, "Effects of refrigerant charge and structural parameters on the performance of a directexpansion solar-assisted heat pump system," Applied Thermal Engineering, vol. 73, no. 1, pp. 522-528, 2014. [Online]. Available: https: //doi.org/10.1016/j.applthermaleng.2014.07.077

[21] G.-H. Shi, L. Aye, D. Li, and X.-J. Du, "Recent advances in direct expansion solar assisted heat pump systems: A review," Renewable and Sustainable Energy Reviews, vol. 109, pp. 349-366, 2019. [Online]. Available: https://doi.org/10.1016/j.rser.2019.04.044

[22] X. Kong, P. Sun, Y. Li, K. Jiang, and S. Dong, "Experimental studies of a variable capacity directexpansion solar-assisted heat pump water heater in autumn and winter conditions," Solar Energy, vol. 170 , pp. $352-357,2018$. [Online]. Available: https://doi.org/10.1016/j.solener.2018.05.081

[23] W. Quitiaquez, I. Simbaña, R. Caizatoa, C. Isaza, C. Nieto, P. Quitiaquez, and F. Toapanta, "Análisis del rendimiento termodinámico de una bomba de calor asistida por energía solar utilizando un condensador con recirculación," Revista Técnica Energía, vol. 16, no. 2, pp. 111-125, 2020. [Online]. Available: https://doi. org/10.37116/revistaenergia.v16.n2.2020.358

[24] G. K. Singh, S. Pradhan, and V. Tanna, "Experimental studies of two phase flow characteristics and void fraction predictions in steady state horizontal two-phase nitrogen flow," Cryogenics, vol. 100, pp. 77-84, 2019. [Online]. Available: https://doi.org/10.1016/j.cryogenics.2019.04.007

[25] C.-M. Yang and P. Hrnjak, "A new flow pattern map for flow boiling of R410a in horizontal micro-fin tubes considering the effect of the helix angle," International Journal of Refrigeration, vol. 109, pp. 154-160, 2020. [Online]. Available: https://doi.org/10.1016/j.ijrefrig.2019.09.013

[26] M. Shafaee, H. Mashouf, A. Sarmadian, and S. Mohseni, "Evaporation heat transfer and pressure drop characteristics of R-600a in horizontal smooth and helically dimpled tubes," Applied Thermal Engineering, vol. 107, pp. 28-36, 2016. [Online]. Available: https: //doi.org/10.1016/j.applthermaleng.2016.06.148 
[27] Z.-Q. Yang, G.-F. Chen, X.-R. Zhuang, Q.-L. Song, Z. Deng, J. Shen, and M.-Q. Gong, "A new flow pattern map for flow boiling of R1234ze(E) in a horizontal tube," International Journal of Multiphase Flow, vol. 98, pp. 24-35, 2018. [Online]. Available: https://doi.org/10.1016/ j.ijmultiphaseflow.2017.08.015

[28] L. Wojtan, T. Ursenbacher, and J. R. Thome, "Investigation of flow boiling in horizontal tubes: Part II-Development of a new heat transfer model for stratified-wavy, dryout and mist flow regimes," International Journal of Heat and Mass Transfer, vol. 48, no. 14, pp. 2970-2985, 2005. [Online]. Available: https://doi.org/10.1016/ j.ijheatmasstransfer.2004.12.013

[29] J. D. de Oliveira, J. C. Passos, J. B. Copetti, and C. van der Geld, "Flow boiling heat transfer of propane in $1.0 \mathrm{~mm}$ tube," Experimental Thermal and Fluid Science, vol. 96, pp. 243-256, 2018. [Online]. Available: https: //doi.org/10.1016/j.expthermflusci.2018.03.010 